\title{
A Role for Long Noncoding RNAs in Implantation Failure
}

\author{
Maria Rosa Maduro, PhD
}

Recurrent implantation failure, defined as the failure to achieve a clinical pregnancy after transfer of at least 4 good quality embryos in a minimum of 3 fresh or frozen cycles in a woman under the age of 40 years old, is unfortunately a common event after in vitro fertilization. In fact, implantation failure is estimated to occur in $60 \%$ to $70 \%$ of all embryo transfers. ${ }^{1}$ Both embryonic and maternal factors contribute to this outcome, in which impaired endometrial receptivity is thought to play a major role. ${ }^{2}$ A clear understanding of what is in the basis of poor endometrial receptivity has been challenging to achieve, despite numerous studies in the field. ${ }^{3-7}$

In the current issue of Reproductive Sciences, Chen and colleagues revisit the topic taking a new and promising approach. ${ }^{8}$ The authors aimed to determine whether long noncoding RNAs (lncRNAs), the class of RNA transcripts with a length of more than 200 nucleotides that has been shown to regulate gene expression at multiple levels and in different systems, impact endometrial gene expression in implantation failure.

In order to do so, Chen et al performed RNA sequencing on secretory phase endometrium from 3 patients with recurrent implantation failure after in vitro fertilization treatment with embryo transfer and 3 control women, using high-throughput sequencing. The authors then compared lncRNA and messenger RNA (mRNA) expression levels for both groups to screen for lncRNAs with potentially important roles in the endometrium. A subset of 10 differentially expressed lncRNAs and 6 mRNAs were selected and validated by quantitative reverse transcription-polymerase chain reaction in 20 patients with recurrent implantation failure and 30 controls, and the target genes of the lncRNAs were predicted through bioinformatics with the aim of identifying the lncRNAs' potential biological roles.

Chen and colleagues were able to identify 1.202 differentially expressed genes in midsecretory phase endometrial tissue between patients with recurrent implantation failure and normal controls, of which 742 were $\operatorname{lncRNAs}$ and 460 were mRNAs. Analysis of the lncRNAs' target genes revealed 148 lncRNAs corresponding to 147 cis-regulated target genes. These genes were determined to be clustered into several pathways, of which the tumor necrosis factor signaling pathway, the toll-like receptor signaling pathway, and the nuclear factor "kappa-light-chain-enhancer" of activated B-cells signaling pathway are of particular relevance.

In sum, the results presented by Chen and colleagues constitute the first report on the role of lncRNAs as regulatory factors influencing endometrial receptivity in women experiencing recurrent implantation failure using RNA sequencing. This study lays the foundation for further investigation on the mechanisms of IncRNAs' action and, moreover, evidences the importance of specific pathways likely to play a role in the development of implantation failure.

\section{References}

1. Coughlan C, Ledger W, Wang Q, et al. Recurrent implantation failure: definition and management. Reprod Biomed Online. 2014;28(1):14-38.

2. Simon A, Laufer N. Assessment and treatment of repeated implantation failure (RIF). J Assist Reprod Genet. 2012;29(11): 1227-1239.

3. Silveira CO, Rezende CP, Ferreira MC, Del Puerto HL, Reis FM. Implantation failure is associated with increased $\alpha$-inhibin and $\beta$-glycan gene expression in secretory phase endometrium: nested case-control study of infertile women undergoing IVF/fresh embryo transfer. Reprod Sci. 2017;24(5):720-725.

4. Heitmann RJ, Weitzel RP, Feng Y, Segars JH, Tisdale JF, Wolff EF. Maternal T regulatory cell depletion impairs embryo implantation which can be corrected with adoptive T regulatory cell transfer. Reprod Sci. 2017;24(7):1014-1024.

5. Celik O, Acet M, Kucuk T, et al. Surgery for benign gynecological disorders improve endometrium receptivity. Reprod Sci. 2017; 24(2):174-192.

6. Yen CF, Liao SK, Huang SJ, et al. Decreased endometrial expression of leukemia inhibitory factor receptor disrupts the STAT3 signaling in adenomyosis during the implantation window. Reprod Sci. 2017;24(8):1176-1186.

7. Ersahin A, Celik O, Acet M, et al. Impact of endometrioma resection on eutopic endometrium metabolite contents: noninvasive evaluation of endometrium receptivity. Reprod Sci. 2017;24(5): 790-795.

8. Chen MY, Liao GD, Zhou B, Kang LN, He YM, Li SW. Genomewide profiling of long noncoding RNA expression patterns in women with repeated implantation failure by RNA sequencing [published online January 1, 2018]. Reprod Sci. 2018: 1933719118756752. doi:10.1177/1933719118756752. 